\title{
Colorimetria e espectroscopia no infravermelho médio em madeira de curupixá frente ao intemperismo artificial com produtos de acabamento
}

\author{
Mid-infrared spectroscopy and colorimetry of curupixá wood: artificial \\ weathering along with finishing products
}

\author{
Robert Rossi Silva de Mesquita ${ }^{\mathrm{I}}$, Marcella Hermida de Paula ${ }^{\mathrm{I}}$ \\ Joaquim Carlos Gonçalez ${ }^{\text {II }}$
}

\begin{abstract}
Resumo
O objetivo do estudo foi investigar alterações de cor e de estruturas químicas da superfície da madeira de curupixá (Micropholis sp.), submetida ao intemperismo artificial, com dois produtos de acabamento. As superfícies das amostras foram tratadas com verniz e stain, e submetidas ao intemperismo artificial por 700 horas de acordo com a norma ASTM G154 (2006). O infravermelho médio com transformada de Fourier (FT-MIR) e o sistema CIELAB (1976) foram utilizados para caracterizar mudanças na estrutura química e colorimétricas da superfície da madeira, respectivamente. Em relação à cor, a madeira de curupixá escureceu com a aplicação dos produtos de acabamento. O intemperismo alterou de forma apreciável a cor da testemunha, e de forma muito apreciável a madeira tratada com os dois produtos de acabamento. A análise de espectroscopia no infravermelho médio mostrou que, em todos os casos, sucedeu a despolimerização da lignina acompanhada da formação de carbonilas, além de queda em suas concentrações devido à lixiviação dos metais pesados. Tudo isso indica que houve degradação dos componentes químicos estruturais da madeira e que não houve influência dos produtos de acabamento no processo de intemperismo.
\end{abstract}

Palavras-chave: Superfície da madeira; Fotodegradação; Verniz; Stain

\begin{abstract}
This study investigates the changes in color and in the chemical structures of the surface of curupixá wood (Micropholis sp.), which was exposed to artificial weathering with two wood finishing products. We treated the sample surfaces with varnish and stain, and then we set them to artificial weathering for 700 hours, in accordance with ASTM G154 (2006). Our team used The Fourier Transform Mean Infrared (FT-MIR) and the CIELAB (1976) system in order to outline changes in the chemical and colorimetric structure of the wood surface, respectively. The color of curupixá wood got darker after applying finishing products. The color variation following the weathering process was appreciable in the control sample and very appreciable in the treated wood sample. The analysis of medium infrared spectroscopy demonstrated the depolymerization of lignin accompanied by the formation of carbonyls in all cases. In addition, mid-infrared displayed a concentration drop due to the heavy metal leaching. These ocurrences reveal degradation in the structural chemical components of the wood, and that the finishing products did not affect the weathering process at all.
\end{abstract}

Keywords: Wood surface; Photodegradation; Varnish; Stain

\footnotetext{
Engenheiro(a) Florestal, Me., Universidade de Brasília, Departamento de Engenharia Florestal, Campus Universitário Darcy Ribeiro, CEP 70910900, Brasília (DF), Brasil. robertflorestal@gmail.com (ORCID: 0000-0003-0367-0256) / marcellahermida@hotmail.com (ORCID: 0000-0002-52069945)

II Engenheiro Florestal, Dr. Professor, Departamento de Engenharia Florestal, Universidade de Brasília, Campus Universitário Darcy Ribeiro, CEP 70910-900, Brasília (DF), Brasil. goncalez@unb.br (ORCID: 0000-0003-1627-0833)
} 


\section{Introdução}

As espécies de madeiras amazônicas vêm sendo muito apreciadas nos últimos anos, devido, principalmente, a sua cor atraente, alta durabilidadee outros fatores. Essas espécies são frequentemente utilizadas em pisos, na produção de compensados, laminados, acabamentos e na indústria moveleira. Por ser um material biológico, a madeira está sujeita a diferentes tipos de degradação (SHANBHAGE; SUNDARARAJ, 2013). Quando a madeira é exposta ao ar livre, ela pode sofrer um processo de desgaste, induzido por fatores como umidade, luz solar, calor e vento: o intemperismo. Assim, a madeira pode ficar em desvantagem quando comparada com seus concorrentes, tais como o aço, plástico e concreto (FABIYI; OGUNLEYE, 2015).

De acordo com Hon (2001), o intemperismo é u ma complexa combinação da ação dos raios solares, da chuva ou da umidade e dos ventos que incidem na superfície da madeira, penetrando apenas 2,0 $\mathrm{mm}$ de profundidade. Com a ação do intemperismo, a superfície da madeira sofre mudanças, o que leva,principalmente, a uma alteração da sua cor e brilho (ROWELL, 2005). De início, ocorre uma mudança de cor, e depois, a perda de suas propriedades mecânicas (GONÇALEZ et al., 2010).

Por mais que a ação do intemperismo seja limitada à superfície da madeira, trata-se de um problema para seus consumidores, pois esse processo, além de modificar o brilho, a cor e a textura da madeira, pode afetar o desempenho dos produtos de proteção aplicados sobre a mesma (ISHIGURI et al., 2003).

Vários estudos sobre o assunto têm sido realizados, especificamente relacionando os efeitos do intemperismo sobre a mudança da cor da madeira (PANDEY, 2005; SILVA; PASTORE; PASTORE JUNIOR, 2007). Porém, pesquisas que associam os efeitos do intemperismo sobre o comportamento químico nas superfícies de madeiras amazônicas ainda são escassas.

É sabido que os processos que ocorrem durante a irradiação são reações físicoquímicas complexas que causam a redução no teor de lignina relacionada com um aumento da concentração de hidratos de carbono (PANDEY, 2005). A irradiação de luz altera os grupos cromóforos encontrados na madeira, formando radicais livres e causando alterações na sua cor (AYADI et al., 2003). A degradação da lignina e de extrativos, seguida por processos de oxidação, resultam em novos grupos químicos e cromórofos (PASTORE; SANTOS; RUBIM, 2004).

Um dos métodos que podem ser utilizados na análise de estruturas e processos químicos que ocorrem durante o processo de intemperismo na madeira é a espectroscopia no infravermelho (MITSUI; TSUCHIKAWA, 2005; DARIE et al., 2011).

As técnicas de espectroscopia no infravermelho (próximo, $14000-4000 \mathrm{~cm}^{-1}$; médio, 4000 $400 \mathrm{~cm}^{-1}$; longo, $400-10 \mathrm{~cm}^{-1}$ ) baseiam-se na absorção de raios infravermelhos por moléculas de frequências específicas, pertencentes a estruturas químicas do material analisado (LUYKX; VAN RUTH, 2008).

Em comparação com o espectro de infravermelho próximo (NIR), o espectro de infravermelho médio (MIR) exibe um elevado grau de resolução espectral, obtendo um resultado eficaz dos picos dos componentes químicos da amostra analisada (WILSON; HOLLAND; POTTER, 1994; ROYCHOUDHURY; HARVEY; MCNEIL, 2006).

A técnica de MIR tem a vantagem de providenciar uma mensuração rápida e simples, que requer o processamento mínimo da amostra antes da análise (SINELLI et al., 2008). A espectroscopia MIR é um dos métodos de análise mais práticos, sendo uma ferramenta adequada para investigar a composição química, bem como a estrutura molecular, grupos funcionais e constituintes de madeiras (FACKLER; SCHWANNINGER, 2012).

Dessa maneira, o objetivo deste estudo foi investigar as alterações de cor e os efeitos do intemperismo nas propriedades químicas da superfície da madeira de curupixá (Micropholis sp.) não tratadas e tratadas com dois produtos de acabamento (verniz e stain), e analisar a eficácia destes. 


\section{Material e métodos}

\section{Origem e confecção dos corpos de prova}

A madeira de Micropholis sp. (curupixá) utilizada neste estudo foi obtida no mercado madeireiro local de Brasília. Foram escolhidas 3 peças de madeira de $1500 \mathrm{~mm}$ x $100 \mathrm{~mm}$ x $25 \mathrm{~mm}$ (comprimento x largura $\mathrm{x}$ espessura, respectivamente) e a partir dessas peças foram confeccionadas 30 amostras, com dimensões de $75 \mathrm{~mm}$ x $30 \mathrm{~mm}$ x $5 \mathrm{~mm}$ (comprimento x largura x espessura, respectivamente), Em cada amostra, a superfície tangencial utilizada para as análises foi aplainada e polida com lixas, sendo reservadas imediatamente em recipiente fechado ao abrigo da luz.

Os produtos de acabamento selecionados foram o verniz isolante para madeiras, incolor, composto por resinas vinílicas e solventes, e o stain impregnante transparente, composto por polímeros, óleos vegetais e hidrocarbonetos, ambos aplicados de acordo com orientações do fabricante. Apesar de possuírem mecanismos de proteção diferentes, os dois possuem duplo filtro solar. Das 30 amostras da espécie, 10 serviram como testemunha (sem acabamento), 10 foram tratadas com verniz e as outras 10 tratadas com stain.

Os ensaios de intemperismo artificial e a análise de colorimetria foram realizados no Laboratório de Tecnologia da Madeira do Departamento de Engenharia Florestal da Universidade de Brasília (UnB). As análises de espectrometria no infravermelho médio foram feitas no Setor de Química, Adesivos e BorrachaNatural do Laboratório de Produtos Florestais (LPF) do Serviço Florestal Brasileiro (SFB).

\section{Ensaio de intemperismo artificial}

Para este ensaio foi utilizada uma câmara de envelhecimento acelerado marca Q-lab modelo QUV/Spray, que simula o processo de intemperismo causado na superfície de madeira. Além disso, foi adotada a norma ASTM G154 de ciclo 7 (2006):cada ciclo é composto por 8 horas de exposição à radiação UV (irradiância de $1.36 \mathrm{~W} / \mathrm{m}^{2}$ a $340 \mathrm{~nm}$ ), 15 minutos de spray de água simulando a precipitação e 3 horas e 45 minutos de condensação de água a $50^{\circ} \mathrm{C}$. O tempo total de intemperismo foi de 700 horas divididas em 7 períodos de acordo com a tabela 1.

\section{Tabela 1 - Descrição de cada período de intemperismo}

Table 1 - Description of each weathering period

\begin{tabular}{lccccccc}
\hline \multicolumn{1}{c}{ Período } & $\mathbf{1}^{\circ}$ & $\mathbf{2}^{\circ}$ & $\mathbf{3}^{\circ}$ & $\mathbf{4}^{\circ}$ & $\mathbf{5}^{\circ}$ & $\mathbf{6}^{\circ}$ & $\mathbf{7}^{\circ}$ \\
\hline Horas de ensaio $(\mathbf{h})$ & 0 & 24 & 48 & 120 & 192 & 396 & 700 \\
Ciclos acumulados & 0 & 2 & 4 & 10 & 16 & 33 & 58,3 \\
Dias acumulados & 0 & 1 & 2 & 5 & 8 & 16,5 & 29,16 \\
\hline
\end{tabular}

Fonte: Autores (2018)

Entre cada período de intemperismo, as amostras foram retiradas da câmara para realização de medições de cor por análise colorimétrica e para obtenção dos espectros por espectroscopia no infravermelho médio. 


\section{Colorimetria da madeira}

Para avaliação da mudança de cor entre cada período de exposição ao intemperismo artificial, foi adotado o sistema CIE $L^{*} a^{*} b^{*} 1976$ que consiste nos parâmetros colorimétricos: luminosidade ou claridade $\left(L^{*}\right)$, coordenadas de cromaticidade $\left(a^{*}\right.$ e $\left.b^{*}\right)$, saturação da cor $(C)$ e o ângulo de tinta $\left(h^{*}\right)$. Esse sistema reproduz a sensibilidade humana de cor. Para a obtenção dos parâmetros colorimétricos, foi utilizado um espectrofotómetro ColorEye XTH, marca $\mathrm{X}$-Rite, nas condições: iluminante D65 e ângulo de $10^{\circ}$ em temperatura ambiente, acoplado a um microcomputador utilizando o software IColorRite. Foram tomadas as medidas dos parâmetros colorimétricos na face tangencial das amostras sendo feita a leitura no sentido das fibras, totalizando 10 medições por amostra entre cada ciclo, conforme metodologia de Gonçalez (1993).

Utilizando uma tabela de cores sugerida por Camargos e Gonçalez (2001), foi possível caracterizar a cor da madeira de curupixá em cada tratamento.Para determinar as variações dos parâmetros colorimétricos, calculou-se a variação total da cor $(\Delta \mathrm{E})$ entre os tratamentos conforme a Equação 1, de acordo com a norma ASTMD 2244 (2009).

$$
\Delta E=\sqrt{\Delta L^{2}+\Delta a^{2}+\Delta b^{2}}
$$

Em que: $\Delta \mathrm{E}=$ variação da cor entre ciclos; $\Delta \mathrm{L}=$ variação da luminosidade entre ciclos; $\Delta \mathrm{a}=$ variação do parâmetro a* (parâmetro colorimétrico do eixo de cores vermelho-verde) entre ciclos; $\Delta \mathrm{b}=$ variação do parâmetro $b^{*}$ (parâmetro colorimétrico do eixo de cores amarelo-azul) entre ciclos.

Objetivando classificar a variação da cor foi usada a Tabela 2, proposta por Hikita, Toyoda e Azuma (2001), baseada em níveis de percepção de cor a olho nu.

\section{Tabela 2 - Classificação da variação total da cor $\left(\Delta E^{*}\right)$ de madeiras}

Table 2 - Classification of total color variation $\left(\Delta \mathrm{E}^{*}\right)$ of wood

\begin{tabular}{lc}
\hline Variação da $\operatorname{cor}\left(\Delta \mathbf{E}^{*}\right)$ & Classificação \\
\hline $\mathbf{0 , 0}-\mathbf{0 , 5}$ & Desprezível \\
$\mathbf{0 , 5}-\mathbf{1 , 5}$ & Ligeiramente perceptível \\
$\mathbf{1 , 5}-\mathbf{3 , 0}$ & Notável \\
$\mathbf{3 , 0}-\mathbf{6 , 0}$ & Apreciável \\
$\mathbf{6 , 0}-\mathbf{1 2 , 0}$ & Muito apreciável \\
\hline
\end{tabular}

Fonte: Hikita, Toyoda e Azuma (2001).

Os valores das variáveis colorimétricas foram obtidos diretamente pelos softwares de aquisição de dados e posteriormente tabulados em planilhas eletrônicas do software EXCEL 2016. Foram feitos testes iniciais que comprovaram a normalidade da distribuição dos dados (Shapiro-Wilk a 95\% de probabilidade), e então foi executada a análise de variância (ANOVA), com auxílio do programa ASSISTAT 7.7, para verificar se existia diferençaestatística entre os parâmetros antes e após a aplicação dos produtos de acabamento dentre os tratamentos. Para os dados nos quais as médias das amostras diferiram estatisticamente entre ciclos de intemperismo, foi aplicado o teste de médias de Tukey a $5 \%$ de significância. 


\section{Espectroscopia no infravermelho médio}

A análise do efeito do intemperismo na composição química da superfície das amostras de curupixá foi determinada utilizando o infravermelho médio com transformada de Fourier(FT-IR). As medições do infravermelho médio foram realizadas em sala climatizada, com temperatura e umidade controladas para evitar interferências na obtenção dos dados. Foi utilizado o dispositivo de reflectância difusa EasyDiff, da marca Pike, acoplado em um espectofotômetro Tensor 37 da marca Bruker. Todas as medidas foram controladas utilizando o software Opus 6.5.

Para cada amostra, foram obtidos 3 espectros em diferentes posições da face tangencial e, em seguida, foi feita a média dos espectros. Cada espectro foi logrado pela média de 64 varreduras com resolução de $4 \mathrm{~cm}^{-1}$, em uma faixa espectral de 4000 a $400 \mathrm{~cm}^{-1}$.

Antes da obtenção de cada espectro, foi utilizado o espelho de alinhamento revestido com monóxido de silício no intuito de obter o sinal de referência (background) para calibração da absorção da luz infravermelha média, eliminando qualquer influência externa.

Após auferir os dados, os espectros foram ajustados utilizando o software Opus 6.5, no qual foi feita a média para cada tratamento, em cada período de intemperismo artificial. A correção da linha de base e os espectros foram normalizados.

Para as comparações quantitativas, os espectros foram normalizados em relação à banda em $899 \mathrm{~cm}^{-1}$, atribuída ao anel de glicose da celulose. As medidas da altura tomadas nessa banda foram feitas nos limites da linha base entre 936 a $866 \mathrm{~cm}^{-1}$.

Foram consideradas para quantificação somente as bandas puras, que são; v(C=O) em 1740 $\mathrm{cm}^{-1}$, referente à concentração de carbonilas, e v(C=C) em $1510 \mathrm{~cm}^{-1}$, referente à concentração de lignina.

\section{Resultados e discussão}

\section{Colorimetria da madeira}

A Figura 1 ilustra os espectros de reflectância difusa visível da madeira de curupixá sem tratamento e da madeira tratada com verniz e stain, antes e após o ensaio de intemperismo artificial, de acordo com o sistema CIELAB 1976. Ao aplicar os produtos de acabamento (verniz e stain), ocorreu o escurecimento da madeira estudada conforme visto na Figura 1, pois se observa a diminuição na intensidade da luz refletida.

Silva, Pastore e Pastore Júnior (2007), em estudo com 5 espécies de madeiras tropicais frente ao intemperismo, empregaram verniz e stain transparente e obtiveram resultado similar, ou seja, a aplicação dos produtos escureceu todas as madeiras estudadas, em função da diminuição na intensidade de luz refletida.

A madeira testemunha antes do processo de intemperismo artificial (T0h) reflete menos a luz em quase todos os comprimentos de onda do espectro quando comparada após o processo de intemperismo (T700h). Para a madeira tratada com verniz, ocorre pouca variação de reflectância nos comprimentos de onda antes e após o tratamento, sendo que para o tratamento com stain essa variação é quase inalterada.

Os valores médios dos parâmetros colorimétricos dos 3 tratamentos durante os ciclos de intemperismo das amostras de curupixá são apresentados na Tabela 3. 


\section{Figura 1 - Curvas de reflectância da madeira de curupixá sem tratamento e das madeiras tratadas com verniz e stain, antes e após o intemperismo acelerado}

Figure 1 - Reflectance curves of untreated curupixá wood versus treated wood with varnish and stain, before and after accelerated weathering

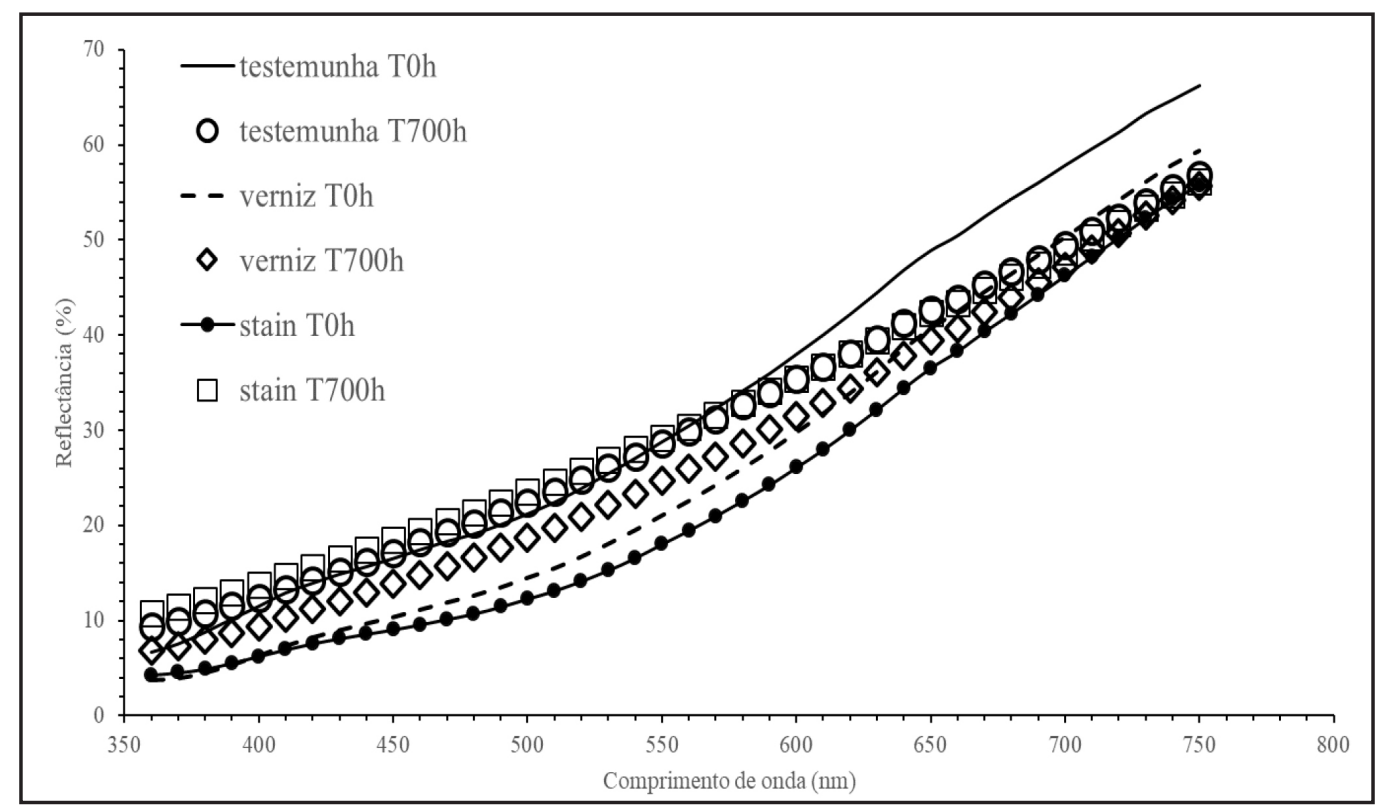

Fonte: Autores (2018)

A cor da madeira de curupixá tem sua formação influenciada pela maior proporção do cromóforo $b^{*}$, indicando a predominância da tonalidade amarela na cor.

De acordo com Camargos e Gonçalez (2001), a madeira é considerada escura para valores de $L^{*}$ (claridade) menores ou iguais a 56 e clara para valores acima de 56 . Dessa forma, podese afirmar que as madeiras que receberam o verniz $(54,08)$ e o stain $(50,76)$ como produtos de acabamento se tornaram mais escuras quando comparadas com a madeira testemunha $(61,32)$, e que as amostras nos 3 casos escureceram ao iniciar o processo de intemperismo. Todas elas voltaram a clarear no período de 192 horas, tendendo ao clareamento ao longo do ensaio, já que quando os valores de luminosidade são próximos de 100,eles se inclinam ao branco absoluto.

As alterações de cor na superfície das amostras ocorrem devido ao crescente aumento de cromóforos durante a irradiação.

Observam-se maiores valores dos parâmetros $a^{*}, b^{*}$ e $C$ para madeiras tratadas com verniz durante o processo de intemperismo, com aumento estatisticamente significativo para esses parâmetros comparados com os parâmetros da madeira sem produtos (testemunha). Antes de iniciar o processo de intemperismo ( $\mathrm{T} \mathrm{h}$ ), observa-se que ao aplicar produtos de acabamento ocorre um aumento nos parâmetros $a^{*}, b^{*}$ e $C$ e redução no parâmetro $L^{*}$. Comportamento semelhante foi observado por Gonçalez et al. (2010), que ao aplicarem os produtos stain e seladora em amostras de freijó (Cordia goeldiana), verificaram uma alteração de cor (escurecimento), seguida de uma redução da claridade $\left(L^{*}\right)$ e variação dos parâmetros $a^{*}$ e $b^{*}$, sugerindo que os produtos aplicados possuem pigmentação ou podem interagir, resultando em formação de pigmentos, alterando a cor superficial da madeira. 


\section{Tabela 3 - Média dos parâmetros colorimétricos da madeira de curupixá sem tratamento (testemunha), e para madeiras tratadas com verniz e stain, para cada período de intemperismo artificial acelerado}

Table 3 - Mean of the colorimetric parameters of the untreated (control) curupixá wood versus the wood treated with varnish and stain for each period of accelerated artificial weathering

\begin{tabular}{|c|c|c|c|c|c|c|}
\hline Tratamento & Tempo (h) & $\mathbf{L}^{*}$ & $a^{*}$ & $\mathbf{b}^{*}$ & $\mathrm{C}$ & $\mathbf{h}^{*}$ \\
\hline \multirow{7}{*}{ Testemunha } & 0 & $61,32 \mathrm{a}$ & $10,87 \mathrm{abc}$ & $23,11 \mathrm{ab}$ & $25,55 a$ & $64,85 a$ \\
\hline & 24 & $55,96 \mathrm{~b}$ & $11,74 \mathrm{a}$ & $26,82 \mathrm{c}$ & $29,29 b$ & $66,40 \mathrm{ab}$ \\
\hline & 48 & $54,82 \mathrm{~b}$ & $11,54 \mathrm{ab}$ & $26,39 \mathrm{~cd}$ & $28,82 \mathrm{bc}$ & $66,43 \mathrm{ab}$ \\
\hline & 120 & $54,10 \mathrm{~b}$ & $10,03 \mathrm{bc}$ & $24,67 \mathrm{ad}$ & $26,64 \mathrm{ac}$ & $67,91 b c$ \\
\hline & 192 & $56,17 \mathrm{~b}$ & $9,52 \mathrm{c}$ & $24,20 \mathrm{a}$ & $26,01 \mathrm{a}$ & $68,54 \mathrm{bc}$ \\
\hline & 396 & $60,12 b$ & $7,90 \mathrm{~d}$ & 21,65 be & $23,06 \mathrm{~d}$ & $69,99 \mathrm{~cd}$ \\
\hline & 700 & $60,62 \mathrm{a}$ & $7,14 \mathrm{~d}$ & $20,89 \mathrm{e}$ & $22,08 \mathrm{~d}$ & $71,25 \mathrm{~d}$ \\
\hline \multirow{7}{*}{ Verniz } & 0 & $54,08 \mathrm{a}$ & $12,62 \mathrm{a}$ & $26,05 \mathrm{a}$ & $28,96 a$ & $64,17 \mathrm{a}$ \\
\hline & 24 & $51,05 b$ & $13,18 \mathrm{a}$ & $28,73 b$ & $31,61 b$ & $65,37 \mathrm{ab}$ \\
\hline & 48 & $49,75 \mathrm{bc}$ & $13,16 \mathrm{a}$ & $28,83 b$ & $31,70 \mathrm{~b}$ & $65,47 a b$ \\
\hline & 120 & $47,98 \mathrm{c}$ & $12,37 \mathrm{a}$ & $27,65 b c$ & $30,30 \mathrm{ab}$ & $65,90 \mathrm{~b}$ \\
\hline & 192 & $49,79 b$ & $12,52 \mathrm{a}$ & $28,16 b$ & $30,83 b$ & $66,04 b c$ \\
\hline & 396 & $53,01 \mathrm{a}$ & $10,93 b$ & $26,28 \mathrm{ac}$ & $28,47 \mathrm{a}$ & $67,48 \mathrm{c}$ \\
\hline & 700 & $57,15 d$ & $8,24 \mathrm{c}$ & $22,23 d$ & $23,72 c$ & $69,74 d$ \\
\hline \multirow{7}{*}{ Stain } & 0 & $50,76 a$ & $13,35 a$ & $24,63 \mathrm{ab}$ & $28,02 \mathrm{ab}$ & $61,59 a$ \\
\hline & 24 & $50,63 a$ & $13,12 \mathrm{a}$ & $26,66 c$ & $29,73 a$ & $63,83 b$ \\
\hline & 48 & $50,54 \mathrm{a}$ & $12,24 \mathrm{a}$ & $26,05 \mathrm{ac}$ & $28,79 a$ & $64,84 \mathrm{bc}$ \\
\hline & 120 & $51,29 \mathrm{a}$ & $10,29 b$ & $23,98 b$ & $26,10 b$ & $66,78 \mathrm{~cd}$ \\
\hline & 192 & $53,60 \mathrm{~b}$ & $10,39 b$ & $24,44 \mathrm{ab}$ & $26,56 b$ & $66,98 \mathrm{~d}$ \\
\hline & 396 & $57,91 \mathrm{c}$ & $7,91 \mathrm{c}$ & $21,29 d$ & $22,72 \mathrm{c}$ & $69,65 \mathrm{e}$ \\
\hline & 700 & $60,97 d$ & $6,59 c$ & $19,05 \mathrm{e}$ & $20,16 \mathrm{~d}$ & $70,98 \mathrm{e}$ \\
\hline
\end{tabular}

Fonte: Autores (2018)

Em que: $L^{*}$ - Luminosidade ou claridade, $\mathrm{a}^{*}$ - Coordenada cromática vermelho-verde, $\mathrm{b}^{*}$ - Coordenada cromática amarelo-vermelho, C - saturação ou cromacidade, $\mathrm{h}^{*}$ - ângulo de tinta, T0h - 0 horas de intemperismo, T24h - 24 horas de intemperismo, T48h - 48 horas de intemperismo, T120h - 120 horas de intemperismo, T192h - $192 \mathrm{horas}$ de intemperismo, T396h - 396 horas de intemperismo, T700h - 700 horas de intemperismo. Valores na mesma coluna para cada tratamento não diferem estatisticamente pelo Teste de Tukey ao nível de $5 \%$ de confiabilidade.

Os parâmetros $\mathrm{L}^{*} \mathrm{e} \mathrm{h}^{*}$ não variaram estatisticamente, mas foram observados maiores valores para a madeira sem produto ao longo do ensaio, possivelmente por esta não receber algum tipo de produto antes do processo de intemperismo. Daniel et al. (2004) afirmam que produtos podem alterar a cor natural da madeira, induzindo a presença de pigmento na composição desses artigos.

Percebe-se que antes do processo de intemperismo (T0h), a madeira tratada com stain possuía coordenadas $a^{*}, b^{*}, C$ elevadas, e $L^{*}$ e $h^{*}$ reduzidas quando comparada com a madeira 
sem produto, então ocorre um aumento nas coordenadas $\mathrm{a}^{*} \mathrm{e} \mathrm{b}^{*}$ após as primeiras 24 horas. Após o período de 192 horas, as coordenadas apresentaram redução, tendendo à estabilização ao final do tratamento, com valores próximos aos da madeira sem exposição ao intemperismo.

O efeito do intemperismo na luminosidade $\left(\mathrm{L}^{*}\right)$, cromaticidade $(C)$ e ângulo de tinta $\left(\mathrm{h}^{*}\right)$ foi correspondente ao observado em relação aos parâmetros $a^{*}$ e $b^{*}$, indicando uma perda de luminosidade nas primeiras 24 horas, com posterior estabilização. Comportamento análogo foi observado em estudos similares: o clareamento de madeiras escuras tende a se estabilizar com o aumento do tempo de intemperismo (SILVA; PASTORE, 2004; SILVA; PASTORE; PASTORE JUNIOR, 2007).

A Tabela 4 apresenta a variação total da cor $(\Delta \mathrm{E})$ para todos os tratamentos durante o experimento.

\section{Tabela 4 - Variação total da cor $(\Delta E)$ para madeira de curupixá sem tratamentoe para madeira tratada com verniz e stain para cada tempo de exposição}

Table 4 - Total color variation $(\Delta \mathrm{E})$ for untreated curupixá wood versus wood treated with varnish and stain for each exposure time

\begin{tabular}{lcccc}
\hline Tratamento & Exposição $(\mathbf{h})$ & $\boldsymbol{\Delta E}$ & Classificação $\left.{ }^{*}\right)$ & Cor $\left.{ }^{* *}\right)$ \\
\hline \multirow{2}{*}{ Testemunha } & T0h & & & Marrom-oliva \\
& T700h & 4,4 & Apreciável & Oliva-amarelado \\
\hline \multirow{2}{*}{ Verniz } & T $0 \mathrm{~h}$ & & & Marrom-oliva \\
& T700h & 6,6 & Muito apreciável & Oliva-amarelado \\
\hline \multirow{2}{*}{ Stain } & T $0 \mathrm{~h}$ & & & Marrom-oliva \\
& T700h & 13,5 & Muito apreciável & Oliva-amarelado \\
\hline
\end{tabular}

Fonte: Autores (2018)

Em que: $\left({ }^{*}\right)$ = Classificação da variação da cor, proposta por Hikita,Toyoda e Azuma, (2001), baseada em níveis de percepção. $\left(^{(*)}\right.$ = Cor da madeira, segundo classificação proposta por Camargos e Gonçalez (2001).

A madeira natural (T0h) foi classificada como marrom-oliva e a simples aplicação dos produtos de acabamento (stain e verniz) não promoveu alteração da cor de acordo com essa classificação. O intemperismo artificial acelerado gerou alteração igual na cor da madeira de curupixá para todos os tratamentos (testemunha, verniz e stain).A cor passou a ser classificada como oliva-amarelado. Essa mudança de cor da madeira de curupixá,após 700 h de intemperismo,é tida como apreciável para a testemunha e muito apreciável quando tratada com os dois produtos de acabamento.

Um dos fatores que explicam a mudança na coloração da madeira é a quantidade de extrativos presentes na parede celular que resulta, no decorrer do tempo, em um escurecimento ou clareamento da madeira quando exposta à luz (CHEN et al., 2012; CADEMARTORI et al., 2014; CONTE et al., 2014).

\section{Espectroscopia no infravermelho}

A espectroscopia de infravermelho médio com transformada de Fourier (FT-MIR) foi utilizada para investigar as mudançasquímicas das amostras que sofreram intemperismo. $\mathrm{O}$ espectro do infravermelho da madeira de curupixá apresenta a mesma estrutura básica de todas as amostras de madeira.

É nítido que a ação do intemperismo conduz alterações significativas tanto na estrutura 
das madeiras sem tratamento, quanto na das madeiras com produtos de acabamento, como observado nas Figuras 2.

\section{Figura 2 - Evolução dos espectros FT-MIR da madeira de curupixá sem tratamento (A), da madeira tratada com verniz $(B)$ e com Stain $(C)$}

Figure 2 - Evolution of FT-MIR spectra of untreated curupixá wood, wood treated with varnish (A) and with stain (B)

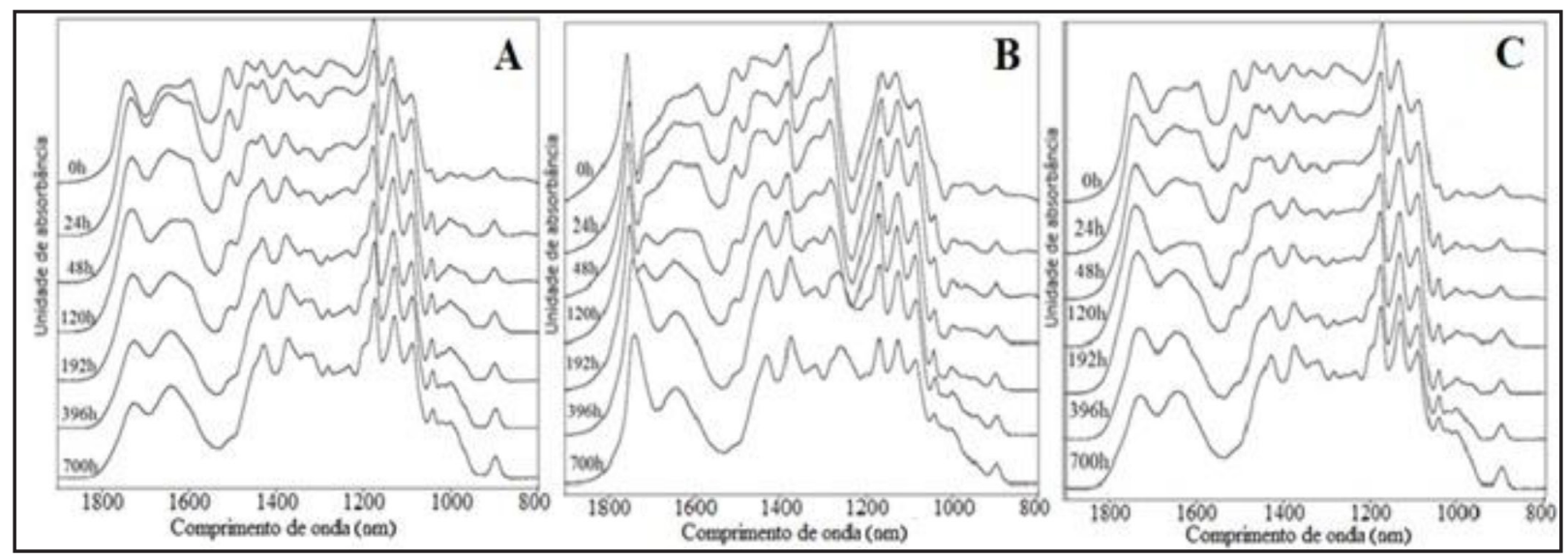

Fonte: Autores (2018)

Observa-se que para todos os tratamentos, durante o processo de intemperismo, a banda de absorção relacionadas a lignina pura, presente no pico $1510 \mathrm{~cm}^{-1}$, diminuiu rapidamente nas primeiras 120 horas de forma gradual, chegando a se exaurir completamente após 700 horas de exposição ao intemperismo.

A Tabela 5 apresenta a variação na intensidade das bandas selecionadas dos espectros da madeira de curupixá sem produto de acabamento, da tratada com verniz e da tratada com stain.

Tabela 5 - Variação na intensidade das bandas de carbonila e lignina dos espectros da madeira de curupixá sem aplicação de produto, da tratada com verniz e da com stain

Table 5 - Variation in the intensity of the carbonyl and lignin bands of the curupixá wood spectra without product application, the one treated with varnish and the other one with stain

\begin{tabular}{ccccccc}
\hline Curupixá & \multicolumn{2}{c}{ Testemunha $\left(\right.$ u.a cm $\left.{ }^{-1}\right)$} & \multicolumn{2}{c}{ Verniz $\left(\mathrm{u} . \mathrm{a} \mathrm{cm}^{-1}\right)$} & \multicolumn{2}{c}{ Stain $\left(\mathrm{u} . \mathrm{cm}^{-1}\right)$} \\
\hline Tempo (h) & carbonila & Lignina & Carbonila & Lignina & carbonila & Lignina \\
\hline 0 & 0,173 & 0,104 & 0,332 & 0,059 & 0,178 & 0,083 \\
24 & 0,147 & 0,086 & 0,244 & 0,063 & 0,165 & 0,062 \\
48 & 0,132 & 0,065 & 0,247 & 0,047 & 0,169 & 0,051 \\
120 & 0,163 & 0 & 0,253 & 0,031 & 0,192 & 0,022 \\
192 & 0,143 & 0 & 0,217 & 0 & 0,173 & 0 \\
396 & 0,115 & 0 & 0,164 & 0 & 0,167 & 0 \\
700 & 0,108 & 0 & 0,131 & 0 & 0,147 & 0 \\
\hline
\end{tabular}

Fonte: Autores (2018)

Em que: u.a: unidade de absorbância. 
Para todos os tratamentos, nota-se que nas primeiras 120 horas de intemperismo ocorre um aumento no teor de carbonilas, com posterior declínio influenciado pelo esgotamento do teor de lignina pura da superfície da madeira. É observada uma concentração maior do teor de carbonila nas amostras tratadas com verniz, evidenciando a presença de compostos carbonílicos na composição do produto de acabamento.

A redução no teor de lignina indica que provavelmente houve sua despolimerização, além de outros processos de degradação. Foram observados fenômenos similares em trabalhos anteriores (ANDERSON et al., 1991; MÜLLER et al., 2003; PASTORE; SANTOS; RUBIM, 2004; XIAOQING; HAIQING, 2008; ZBOROWSKA et al., 2016), nos quais a lignina é o principal constituinte susceptível à fotodegradação.

O fator considerado mais prejudicial é a radiação ultravioleta - UV (30-40 nm) do espectro solar, pois provoca reações de despolimerização da lignina (FEIST; HON, 1984).

A lignina é responsável pela absorção de 80 a 95\% do total de luz UV absorvida pela madeira, hidratos de carbono de 5 a 20\% e extrativos cerca de 2\% (NORRSTROM; HARTLER, 1969).

Em estudo similar, Rosu et al. (2010) afirmam que a absorção em $1510 \mathrm{~cm}^{-1}$ decorre da vibração do esqueleto de anel aromático de benzeno, característico da lignina. O decréscimo na intensidade desse pico indica a degradação da lignina durante o processo de irradiação.

A lignina degradada está presente nos grupos cromóforos que são capazes de absorver a radiação de luz UV entre os comprimentos de onda com posterior formação de radicais livres (MOORE; OWEN, 2001).

A degradação das estruturas aromáticas das ligninas resulta na formação de grupos não conjugados carbonílicos (DIRCKX et al., 1992; ZBOROWSKA et al., 2016). Esses grupos por sua vez reagem com o oxigênio, produzindo grupos carbonilo e carbóxilo, que são relacionados com a descoloração da madeira por ocorrência de compostos de carbonilos saturados (quinonas). O declínio do teor de carbonilas ocorre devido à eliminação de compostos de baixo peso molecular, contendo grupos carbonílicos e carboxílicos que são facilmente removíveis pela água (PASTORE; SANTOS; RUBIM, 2004). Com a lixiviação dos compostos de baixo peso molecular, a madeira se torna mais áspera e esbranquiçada, resultando em um aumento na proporcionalidade de celulose existente na madeira em comparação com a lignina, aumentando a luminosidade (HON, 2001).

Com base nos resultados de espectroscopia, é observado que o mesmo comportamento ocorreu em todos os tratamentos, indicando que não houve influência do produto de acabamento para a madeira de curupixá, exceto para as primeiras 120 horas, quando ainda havia concentrações de lignina para as madeiras tratadas com verniz e stain.

\section{Conclusão}

Foram evidenciadas, de maneira similar, alterações nas propriedades colorimétricas e químicas da superfície da madeira de curupixá sem tratamento, da tratada com verniz e da tratada com stain, quando submetidas ao ensaio de intemperismo artificial. Como efeitos da fotodegradação, além da variação apreciável na cor das amostras, também observa-se que houve a despolimerização da lignina e lixiviação dos compostos químicos da madeira, indicando que a aplicação de verniz e stain não foi suficientepara evitar a deterioração da madeira pelo intemperismo.

\section{Referências}

AMERICAN SOCIETY FOR TESTING AND MATERIALS. ASTM G 154 - Standard Practice for Operating Fluorescent Light Apparatus for UV Exposure of Nonmetallic Materials. Philadelphia, 2006. 
AMERICAN SOCIETY FOR TESTING AND MATERIALS. ASTM D2244 - Standard Practice for Calculation of Color Tolerances and Color Differences from Instrumentally Measured Color Coordinates. Philadelphia, 2009.

ANDERSON, E. L. et al. Infrared studies of wood weathering. Part I: Softwoods. Applied spectroscopy, Washington, v. 45, n. 4, p. 641-647, 1991.

AYADI, N. et al. Color stability of heat-treated wood during artificial weathering. Holz als Rohund Werkstoff, [s. 1.], v. 61, n. 3, p. 221-226, 2003.

CADEMARTORI, P. H. G. D. et al. Colour responses of two fast-growing hardwoods to two-step steam-heat treatments. Materials Research, [s. 1.], v. 17, n. 2, p. 487-493, 2014.

CAMARGOS, J. A. A.; GONÇALEZ, J. C. A colorimetria aplicada como instrumento na elaboração de uma tabela de cores de madeira. Brasil Florestal, [s. 1.], n. 71, p. 30-41, 2001.

CHEN, Y. et al. The effect of heat treatment on the chemical and color change of black locust (Robinia pseudoacacia) wood flour. BioResources, v. 7, n. 1, p. 1157-1170, 2012.

CONTE, B. et al. Propriedades físicas e colorimétricas da madeira termorretificada de Pinus elliottii var. elliottii. Scientia Forestalis, Piracicaba, v. 42, n. 104, p. 555-563, 2014.

DANIEL, T. et al. Clear exterior finishes: finding the balance between aesthetics and durability. JCT CoatingsTech, [s. 1.], v. 1, n. 9, p. 42-48, 2004.

DARIE, R. N. et al. Evaluation of properties of LDPE/oak wood composites exposed to artificial ageing. Cellulose Chemical Technology, Ghica Voda, v. 45, n. 1-2, p. 127-135, 2011.

DIRCKX, O. et al. Modifications de lacouleurdu bois d'Abiesgrandis exposé à lalumièresolaire. Annales des Sciences Forestières, [s. 1.], p. 425-447, 1992

FABIYI, J. S.; OGUNLEYE, B. M. Mid-infrared spectroscopy and dynamic mechanical analysis of heat-treated obeche (Triplochiton scleroxylon) wood. Maderas: Ciencia y tecnología, Concepción, v. 17, n. 1, p. 05-16, 2015.

FACKLER, K.; SCHWANNINGER, M. How spectroscopy and microspectroscopy of degraded wood contribute to understand fungal wood decay. Applied microbiology and biotechnology, [s. 1.], v. 96, n. 3, p. 587-599, 2012.

FEIST, W. C.; HON, D. N. S. Chemistry of weathering e protection. In: ROWELL, R. M. The chemistry of solid wood. Washington: American Chemical Society, 1984. 614 p.

GONÇALEZ, J. C. Caracterisation technologique de quatre espéces peu connues da $\mathbf{1}$ aforêt Amazonienne: anatomie, chimie, couleur, propriétésphysiques et mécaniques. Nancy. 1993. Thèse (Doctorat) - Ecole Nationale du Génie Rural des Eaux et des Forêts, Nancy, 1993.

GONÇALEZ, J. C. et al. Efeito da radiação ultravioleta na cor da madeira de freijó (Cordiagoeldiana Huber) após receber produtos de acabamentos. Ciência Florestal, Santa Maria, v. 20, n. 4, p. 657$664,2010$.

HIKITA, Y.; TOYODA, T.; AZUMA, M. Weathering testing of timber: discoloration.In: IMAMURA, Y. High performance utilization of wood for outdoor uses. Kyoto: Press-Net, 2001.

HON, D. N. S. Weathering and photochemistry of wood. In: HON, D. N. S.; SHIRAISHI, N. Wood and cellulosic chemistry. 2. ed. New York: Marcel Dekker, 2001. v. 2. p. 512-546.

ISHIGURI, F. et al. Extractives relating to heartwood color changes in sugi (Cryptomeria japonica) by a combination of smoke-heating and UV radiation exposure. Journal of wood science, [s. 1.], v. 49 , n. 2 , p. 135-139, 2003.

LUYKX, D. M.; VAN RUTH, S. M. An overview of analytical methods for determining the geographical origin of food products. Food Chemistry, Norwich, v. 107, n. 2, p. 897-911, 2008. 
MITSUI, K.; TSUCHIKAWA, S. Low atmospheric temperature dependence on photodegradation of wood. Journal of Photochemistry and photobiology B: Biology, [s. 1.], v. 81, n. 2, p. 84-88, 2005.

MOORE, A. K.; OWEN, N. L. Infrared spectroscopic studies of solid wood. Applied Spectroscopy Reviews, [s. 1.], v. 36, n. 1, p. 65-86, 2001.

MÜLLER, U. et al. Yellowing and IR-changes of spruce wood as result of UV-irradiation. Journal of Photochemistry and Photobiology B: Biology, [s. 1.], v. 69, n. 2, p. 97-105, 2003.

NORRSTROM, H.; HARTLER, N. Light absorbing properties of pulp and pulp components. Svensk Pappers Institute, Stockholm, v. 72, n. 9, p. 25-38, 1969.

PANDEY, K. K. A note on the influence of extractives on the photo-discoloration and photodegradation of wood. Polymer degradation and stability, [s. 1.], v. 87, n. 2, p. 375-379, 2005.

PASTORE, T. C.; SANTOS, K. O.; RUBIM, J. C. A spectrocolorimetric study on the effect of ultraviolet irradiation of four tropical hardwoods. Bioresource technology, EUA, v. 93, n. 1, p. 37-42, 2004.

ROSU, D. et al. FTIR and color change of the modified wood as a result of artificial light irradiation. Journal of Photochemistry and Photobiology B: Biology, [s. 1.], v. 99, n. 3, p. 144$149,2010$.

ROYCHOUDHURY, P.; HARVEY, L. M.; MCNEIL, B. The potential of mid infrared spectroscopy (MIRS) for real time bioprocess monitoring. Analytica Chimica Acta, Netherlands, v. 571, n. 2, p. $159-166,2006$.

ROWELL, R. M. Handbook of wood chemistry and wood composites. Boca Raton: Taylor and Francis, 2005.

SHANBHAG, R. R.; SUNDARARAJ, R. Physical and chemical properties of some imported woods and their degradation by termites. Journal of Insect Science, Oxford, v. 13, n. 1, p. 63, 2013.

SILVA, J. O.; PASTORE, T. C. M. Fotodecomposição e proteção de madeiras tropicais. Floresta e Ambiente, Seropédica, v. 11, n. 2, p. 07-13, 2004.

SILVA, J. O.; PASTORE, T. C. M.; PASTORE JÚNIOR, F. Resistência ao intemperismo artificial de cinco madeiras tropicais e de dois produtos de acabamento. Ciência Florestal, Santa Maria, v. 17 , n. 1, p. 17-23, 2007.

SINELLI, N. et al. Evaluation of quality and nutraceutical content of blueberries (Vaccinium corymbosum L.) by near and mid-infrared spectroscopy. Postharvest Biology and Technology, Netherlands, v. 50, n. 1, p. 31-36, 2008.

XIAOQING, W., HAIQING, R. Comparative study of the photo-discoloration of moso bamboo (Phyllostachys pubescens Mazel) and two wood species. Applied Surface Science, [s. l.], v. 259, p. 7029-7034, 2008.

ZBOROWSKA, M. et al. Colourimetric and FT-IR ATR spectroscopy studies of degradative effects of ultraviolet light on the surface of exotic ipe (Tabebuia sp.) wood. Cellulose Chemistry and Technology, Romanian, v. 50, p. 71-76, 2016.

WILSON, R. H.; HOLLAND, J. K.; POTTER, J. Lining up for FTIR analysis. Chemistry in Britain, UK, v. 30, n. 12, p. 993-6, 1994. 